\title{
Another way logic might be normative
}

\section{J. W. Evershed ${ }^{1}$}

Received: 17 February 2020 / Accepted: 19 January 2021 / Published online: 21 February 2021

(c) The Author(s) 2021, corrected publication 2021

\begin{abstract}
Is logic normative for reasoning? In the wake of work by Gilbert Harman and John MacFarlane, this question has been reduced to: are there any adequate bridge principles which link logical facts to normative constraints on reasoning? Hitherto, defenders of the normativity of logic have exclusively focussed on identifying adequate validity bridge principles: principles linking validity facts - facts of the form $\Gamma \models \varphi$ - to normative constraints on reasoning. This paper argues for two claims. First, for the time being at least, Harman's challenge cannot be surmounted by articulating validity bridge principles. Second, Harman's challenge can be met by articulating invalidity bridge principles: principles linking invalidity facts of the form $\Gamma \forall \psi$ to normative constraints on reasoning. In doing so, I provide a novel defence of the normativity of logic.
\end{abstract}

Keywords Philosophy of logic $\cdot$ Normativity of logic $\cdot$ Bridge principles $\cdot$ Logic and reasoning

\section{Introduction}

A time-honoured tradition has it that logic is normative. In Kant's words, "In Logic we do not want to know how the understanding is and thinks, and how it has hitherto proceeded in thinking, but how it ought to proceed in thinking" (Kant 1885, p. 4). The intuitive pull of this view is clear: agents who have inconsistent beliefs or who fail to believe their beliefs' obvious logical consequences 'go wrong' in some sense. Let us call the claim that logic is normative the normativity thesis and those who endorse it traditionalists.

This paper defends the normativity thesis from the sceptical challenge advanced by Gilbert Harman, according to which there is no substantive connexion between logical facts and normative constraints on reasoning. Existing responses to Harman's chal-

\footnotetext{
$凶 \quad$ J. W. Evershed

jwevershed@gmail.com

1 Department of Philosophy, University of St Andrews, St Andrews, UK
} 
lenge have sought to identify adequate validity bridge principles-principles linking validity facts of the form $\Gamma \models \varphi$ to normative constraints on reasoning. ${ }^{1}$ Although I contend these responses fall short, I too will defend the normativity thesis from Harman's challenge by articulating bridge principles. What is novel about my defence, however, is that the bridge principles I endorse are of a different ilk to those defended elsewhere: they link invalidity facts of the form $\Gamma \not \forall \psi$ to normative constraints on reasoning.

Here is the plan. Section 2 clarifies the normativity thesis and Harman's challenge, before highlighting that Harman's challenge can be surmounted from either of two nonmutually exclusive directions: by articulating adequate validity bridge principles, or by articulating adequate invalidity bridge principles. Section 3 recapitulates the debate regarding validity bridge principles and argues that, despite recent advances, we are not presently in a position to articulate a validity bridge principle equal to Harman's challenge. The rest of the paper argues that Harman's challenge can be surmounted by appeal to invalidity bridge principles. Section 4 introduces a blueprint for invalidity bridge principles, thereby enabling us to map out all their possible permutations. Section 5 articulates the criteria relevant to the evaluation of these permutations' adequacy, whilst Sects. 6 and 7 apply these adequacy criteria to the space of invalidity bridge principles. I conclude that there are adequate and substantive invalidity bridge principles, thereby defending the normativity thesis from Harman's challenge.

\section{Harman's sceptical challenge}

We begin by clarifying the normativity thesis. In particular, when traditionalists assert logic is normative, what is it that they are asserting logic is normative for? Following much of the existing literature, I assume that the answer to this question is reasoning: the formation and revision of our doxastic attitudes via inference (Harman 1984, p. 107). ${ }^{2}$ For example, suppose I know there is either a sifaka or an indri in a tree and, upon catching a glimpse of it, I realise it is too small to be an indri. If I then infer there is a sifaka in the tree, I thereby form a belief via reasoning. Moreover, if per the normativity thesis logic is normative for reasoning, I ought to have reasoned as I did.

The normativity thesis has long been part of logical orthodoxy. ${ }^{3}$ However, the traditionalists' dogmatic slumbers were interrupted by Gilbert Harman's issuance of his sceptical challenge (Harman 1984, 1986). Harman's challenge begins by distinguishing deductive logic from a theory of reasoning, which tells us how we ought to reason, on the grounds that the two have distinct subject-matters. Deductive logic concerns logical facts which delineate static and non-psychological relations between propositions, whereas a theory of reasoning consists of normative constraints concerning how

\footnotetext{
1 Throughout I use $\Gamma$ to refer to a set of propositions, and $\varphi$ and $\psi$ to refer to individual propositions (or whatever one takes the relata of the logical consequence relation to be).

2 One alternative proposal is that logic is normative for public practices such as assertion and debate-see Dogramaci (2015) and Dutilh-Novaes (2015).

3 Historical traditionalists include Aristotle (cf., Smith 1995, p. 27), Kant (1787), and Frege (1893). Contemporary proponents include Beall and Restall (2006), Field (2009), MacFarlane (2004), Milne (2009), Priest (1979), Steinberger (2019a, b, c), and Streumer (2007).
} 
agents ought to form and revise their beliefs (Harman 1984, p. 107; 1986, pp. 3-4). Take modus ponens, the logical fact that $(\varphi \supset \psi) \wedge \varphi \models \psi$. This cannot be a constraint on reasoning as it makes no mention of beliefs-let alone anything about how one ought to form and revise them, such as that if one believes $\varphi \supset \psi$ and $\varphi$ then one ought to believe $\psi$. Thus, the normativity thesis cannot hold simply because logical facts (or statements thereof) are themselves normative constraints on reasoning.

Disabused of the illusion that deductive logic and a theory of reasoning are identical, a gap opens up between the two. In light of this, for the normativity thesis to hold, there must be principles — which MacFarlane (2004) has termed bridge principles_linking logical facts to the normative constraints on reasoning they induce. ${ }^{4}$ Moreover, not just any old bridge principle will do: it must be adequate, where adequacy is defined relative to a set of criteria (these criteria are delineated in the following section as no details are presently required). Since neither of the candidate bridge principles that Harman considers satisfies all the adequacy criteria, he concludes that the normativity thesis is false (1986, p. 20). ${ }^{5}$

In response to Harman's challenge, most traditionalists have tried to transform his modus tollens into a modus ponens by arguing that there are bridge principles other than the two he considers, some of which are adequate. ${ }^{6}$ Since bridge principles simply link logical facts to normative constraints on reasoning, this response may in principle take either of two forms. First, one may try to articulate adequate validity bridge principles linking validity facts-facts of the form $\Gamma \models \varphi$ - to normative constraints on reasoning. ${ }^{7}$ Second, one may argue that there are adequate invalidity bridge principles linking invalidity facts-facts of the form $\Gamma \not \psi$ - to normative constraints on reasoning. Despite this, traditionalists have focussed exclusively on validity bridge principles. ${ }^{8}$ The next section argues that, as of now, this strategy is unable to succeed and therefore Harman's challenge remains unmet.

\section{Validity bridge principles and their discontents}

This section reviews the existing responses to Harman's challenge which attempt to surmount it by articulating adequate validity bridge principles. I argue that, for the

\footnotetext{
4 Traditionalists claim that logical facts induce normative constraints on reasoning in the sense that it is because $\Gamma \models \varphi$ that there are certain constraints on our combinations of beliefs vis-à-vis $\Gamma$ and $\varphi$. A bridge principle is true or adequate iff the logical fact and normative constraint which it links are such that it is in virtue of the former that the latter obtains.

5 Russell (2020) denies that adequate bridge principles suffice for logic to be normative. For logic to be normative in her sense, logical facts must induce normative constraints on reasoning without recourse to normative assumptions external to logic. However, Russell's question and the question concerning whether there are any adequate bridge principles cut across one another. As Russell (2020, pp. 380-381) acknowledges, logic can be normatively inert in her sense despite there being adequate bridge principles. This paper focusses entirely on bridge principles.

6 The alternative is to challenge the distinction on which the major premiss depends-for details of how such a response might go, see Steinberger (2019a, pp. 308-309).

7 MacFarlane (2004), Field (2009), Steinberger (2019a,c), and Streumer (2007) all approach Harman's challenge from this direction.

8 Steinberger (2019b, p. 5) acknowledges the existence of invalidity (or, as he calls them, commissive) bridge principles but does not explore whether they can be used to meet Harman's challenge.
} 
time being, Harman's challenge cannot be surmounted from this direction because a necessary condition for this strategy's success does not hold at present. In doing so, I hope to provide both the requisite background and the motivation for approaching Harman's challenge from the direction of invalidity bridge principles. One point worth emphasising from the outset is that this section is not arguing that there cannot be an adequate validity bridge principle simpliciter; rather, it argues for the weaker claim that we are presently unable to articulate such a principle.

We begin with the seminal work of MacFarlane (2004). The importance of MacFarlane's contribution resides in his methodology for addressing the question of whether there are any adequate validity bridge principles. Broadly speaking, he proceeds in three stages. First, a blueprint for validity bridge principles is formulated, thereby enabling the space of all the possible permutations to be mapped out. Second, the set of criteria that any validity bridge principle must satisfy to qualify as adequate are identified. Finally, these criteria are applied to the various validity bridge principles and conclusions are drawn about their adequacy.

Following Steinberger (2019a, p. 312), MacFarlane's blueprint can be written as:

$V B P:$ If $\delta(\Gamma \models \varphi)$, then $D(\alpha(\Gamma), \beta(\varphi))$.

Its antecedent states a validity fact or an agent's doxastic attitude towards such a fact, where $\delta$ is a variable denoting said attitude ( $\delta$ is empty when the antecedent is factual). $V B P$ 's consequent is a normative constraint on the agent's doxastic attitudes, where $D$ is a deontic operator, and $\alpha$ and $\beta$ are the potentially distinct attitudes being constrained. The relation between the two is that the antecedent is a triggering condition specifying when agents are subject to the normative constraint in the consequent.

Different validity bridge principles can then be generated by varying the values taken by $V B P$ 's parameters:

1. $\delta$ : Does the validity bridge principle have a factual antecedent in which $\delta$ is empty, or an attitudinal antecedent where $\delta$ denotes a doxastic attitude? That is, are agents subject to the normative constraints induced by the validity facts which actually obtain, or those that they think obtain? ${ }^{9}$

2. $\alpha, \beta$ : Do $\alpha$ and $\beta$ refer to agents' beliefs or their degrees of belief? That is, does the normative constraint govern agents' beliefs or their degrees of belief?

3. $D$ : What kind of deontic operator features in the normative constraint? Is it the indefeasible 'ought to' operator, the defeasible 'has reason to' operator, or the even weaker 'permitted to' operator?

4. D's Scope: The normative constraint in $V B P$ 's consequent is typically a conditional of the form $\alpha(\Gamma) \supset \beta(\varphi)$. What scope does the deontic operator take with respect to this conditional? Does it embed into both the antecedent and consequent, just the consequent, or does it take wide scope over the entire conditional?

5. Polarity: Finally, does the normative constraint in the validity bridge principle's consequent positively require that agents hold certain doxastic attitudes, or does it negatively preclude them from holding specific doxastic attitudes?

$\overline{9}$ I use 'think' as a placeholder for any of the doxastic attitudes to which $\delta$ may refer. 
All in all, the variation of these parameters generates a space of seventy-two distinct validity bridge principles. With the first step of MacFarlane's method complete, we move to the second stage of articulating the adequacy criteria. (MacFarlane 2004, pp. 9-12) endorses the following seven ${ }^{10}$ :

(1) Belief Revision. An adequate validity bridge principle must allow agents to revise their initial belief set when it entails an absurdity rather than requiring that they believe the absurdity. Belief Revision therefore precludes an adequate validity bridge principle's deontic operator from solely embedding into the normative constraint's consequent. The resulting constraint-if $s$ believes $\Gamma$ then $s$ ought to believe $\varphi-$ requires an agent to believe all their belief set's consequences, even if they are absurd, and does not give them the option to revise their initial belief set. ${ }^{11}$

(2) Excessive Demands. An adequate validity bridge principle cannot require cognitively limited agents to believe all the logical consequences of their beliefs, since the deduction of many of these consequences is extremely complex and lies beyond their computational limitations. Thus, Excessive Demands requires validity bridge principles to have attitudinal antecedents since this ensures that agents are only bound by the normative constraints induced by the validity facts that they think obtain.

(3) Preface Paradox. In Makinson's (1965) Preface Paradox, an author has meticulously researched every sentence in her latest non-fiction book. Thus, given her evidence, she is rationally required to believe that each individual sentence is true. However, since there is strong inductive evidence that she is fallible and has made an error somewhere, she is also rationally required to believe the negation of the conjunction of all the sentences in the book. Given that the author is rationally required to believe that each individual sentence is true but that their conjunction false, an adequate validity bridge principle should not require that she forgo either belief, despite their inconsistency. Thus, to satisfy Preface Paradox, a validity bridge principle cannot employ the indefeasible 'ought to' operator. ${ }^{12}$

(4) Clutter avoidance. An adequate validity bridge principle cannot require agents to believe all the logical consequences of their beliefs because doing so would clutter their minds with irrelevancies. For instance, my belief that 'The Pope is bald' entails 'The Pope is bald or six standard pieces of Lego can be combined in over 900 million ways', and many other useless disjunctions besides. Despite being easily deducible from my initial belief, I should not be required to believe them all as doing so would needlessly clutter my mind. This can be avoided by supplementing VBP's antecedent with the clause 'and $s$ has reason to consider $\varphi$ ' so that agents are only subject to the normative constraints induced by the validity facts which are relevant to them.

(5) Priority question. An adequate validity bridge principle must respect the fact that the normative constraints induced by validity facts constrain our reasoning irrespective of whether or not we think that said facts obtain. According to MacFarlane,

\footnotetext{
10 Criteria (1)-(4) are found in Harman (1986, Ch. 2) and MacFarlane (2004, p. 12) introduces (5)-(7).

11 An alternative is to endorse a bridge principle employing the defeasible 'reason to' operator (e.g., Pinder 2017). Most commentators have eschewed this option because it violates Strictness (see below), and because a more attractive view is that an agent is required to revise their initial belief set when it entails an absurdity rather than having a reason to believe the absurdity.

12 Alternatively, one might advocate a bridge principle constraining degrees of belief as Field (2009) does. However, as we will see later on, such a bridge principle cannot meet Harman's challenge.
} 
saying otherwise puts the cart before the horse because, if we were only bound by the constraints induced by the logical facts of which we are aware, it follows that:

The more ignorant we are of what follows logically from what, the freer we are to believe whatever we please-however logically incoherent it is. But this looks backwards. We seek logical knowledge so that we will know how we ought to revise our beliefs: not just how we will be obligated to revise them when we acquire this logical knowledge, but how we are obligated to revise them even now, in our state of ignorance (MacFarlane 2004, p. 12).

Since bridge principles' antecedents delineate when agents are subject to the normative constraints on reasoning given in their consequents, Priority Question requires that their antecedents be factual.

(6) Strictness. At least when it comes to the straightforward consequences of agents' belief sets, an adequate validity bridge principle must indefeasibly require agents to either believe said consequences or else forgo at least one of their initial beliefs. As such, Strictness captures the thought that there is something awry with $s$ ' reasoning if $s$ believes $\varphi$ and $\psi$ whilst disbelieving their conjunction, irrespective of any reasons that $s$ has for doing so. Therefore, Strictness demands that validity bridge principles employ the indefeasible 'ought to' operator.

(7) Obtuseness. The normative constraint on reasoning given in an adequate validity bridge principle's consequent must not merely forbid an agent who believes $\varphi$ and $\psi$ from disbelieving $\varphi \wedge \psi$, but must positively require that they believe $\varphi \wedge \psi$. With the adequacy criteria formulated, we turn to step three of MacFarlane's method: ascertaining whether any of the validity bridge principles identified in step one satisfy all the adequacy criteria. Each criterion precludes at least one of a validity bridge principle's parameters from taking certain values-for instance, Strictness precludes a validity bridge principle's deontic operator from taking any value other than 'ought to'. As such, each criterion partitions the space of validity bridge principles into two cells comprised of those validity bridge principles which satisfy it and those which do not. We may think of the application of a criterion to the space of validity bridge principles as eliminating the portion of the space coinciding with the latter cell. The question of whether there are any adequate validity bridge principles can therefore be restated as: once all the adequacy criteria have been applied to the space of validity bridge principles, which part, if any, remains uneliminated?

It is here that MacFarlane comes unstuck. Once all the criteria have been applied, no part remains uneliminated: there are no validity bridge principles satisfying all the criteria (MacFarlane 2004, pp. 12-13). The reason for this is simple. If two adequacy criteria conflict - that is, each demands that one of the parameters takes different values - then no validity bridge principle can satisfy both criteria. Consequently, any conflicting adequacy criteria will together eliminate the entirety of the space of validity bridge principles as inadequate. Thus, we have the following necessary condition for a space of bridge principles, $S$, to contain an adequate member:

Conflict-Free*: None of the adequacy criteria pertaining to $S$ conflict. ${ }^{13}$

13 I have added an asterisk to distinguish this condition from the adequacy criteria. 
Yet, despite MacFarlane's adequacy criteria being fairly weak, there are two separate conflicts amongst them. First, between Excessive Demands and Priority Question: whereas Excessive Demands requires validity bridge principles to have attitudinal antecedents, Priority Question demands that their antecedents be factual. Second, between Strictness and Preface Paradox: Strictness favours principles featuring the indefeasible 'ought to' operator but Preface Paradox pulls in the opposite direction.

This is where the work of Steinberger (2019a,c) enters the fray. Steinberger progresses the debate by highlighting that bridge principles are intended to be norms, and there are three kinds of norm they may be. Using this tripartite distinction, both the nature of Harman's challenge and which adequacy criteria are relevant to a bridge principle's adequacy can be clarified.

Steinberger (2019c, p. 15) begins with the following norm schema:

Norm: If $C$, then $s$ ought to/may/has reason to $\phi$.

$C$ is a triggering condition specifying the conditions under which the agent is subject to the normative constraint governing the action, $\phi$, stated in the consequent. Validity bridge principles, as specified by $V B P$, satisfy this schema. A validity bridge principle's antecedent - a validity fact, or an agent thinking that a validity fact obtains — serves as a triggering condition specifying when an agent's reasoning is subject to the normative constraint on belief combinations stated in its consequent. Steinberger (2019c, p. 16) then distinguishes between three functionally distinct kinds of norms that a validity bridge principle might be:

(1) Evaluations Evaluative norms are objective and ideal standards of correctness. Thus, evaluative validity bridge principles determine, from an objective and ideal standpoint, whether our reasoning is correct or incorrect.

(2) Directives Directive norms guide ordinary agents' actions. Directive validity bridge principles therefore provide us with guidance on how to reason.

(3) Appraisals Appraisal norms are standards against which an ordinary agent's conduct may be praised or criticised. As such, appraisal validity bridge principles determine when our reasoning is logically reprehensible or praiseworthy.

Accordingly, there is not one space of validity bridge principles but three, each comprised of the same principles but differentiated by their normative functions.

This tripartite distinction progresses the debate in two ways. First, it enables traditionalists to clarify Harman's challenge and thereby know exactly what is required to respond to it. Does Harman think logic is not normative for reasoning because it fails to provide evaluative norms, directive norms, appraisal norms, or all three? Harman conceives of a theory of reasoning as providing prescriptions for how agents ought to form and revise their beliefs. As such, he is best understood as asserting that logic is not normative in the sense that it does not provide directive norms for reasoning (Steinberger 2019a, pp. 317-318; 2019c, pp. 27-28). This is evinced by Harman's emphasis on rules that agents could follow when characterising his stance that logic is not normative: 
Deductive rules are rules of implication, not rules of inference or reasoning. They are perhaps rules about what makes something a proof or argument, but they are not rules that you could follow in constructing a proof or argument (Harman 2010, p. 152).

Thus, meeting Harman's challenge requires an adequate directive bridge principle. ${ }^{14}$

The second way that the tripartite distinction progresses the debate is by showing that, owing to their differing functions, different sets of adequacy criteria apply to the three spaces of validity bridge principles (Steinberger 2019c, p. 23). The hope is that disambiguating the three spaces will enable Conflict-Free* to be met for each of them since some of the criteria that were previously thought to conflict do not apply to the same spaces. Steinberger argues that together these clarifications enable Harman's challenge to be met. The remainder of this section disputes this. Although Harman's challenge requires the articulation of an adequate directive bridge principle, the space of directive validity bridge principles does not presently satisfy Conflict-Free*. Thus, as of now, we are unable to articulate an adequate directive validity bridge principle.

Recall that there are two conflicts between MacFarlane's adequacy criteria. First, between Excessive Demands and Priority Question, and second, between Strictness and Preface Paradox. The tripartite distinction enables Steinberger to dissolve the first conflict since Priority Question does not apply to the space of directive validity bridge principles. The reason for this is that Priority Question requires validity bridge principles to capture what logic demands of us as opposed to what we think it demands of us. And whilst this is appropriate for evaluative validity bridge principles which state objective standards for reasoning, it is inappropriate for directive validity bridge principles which, in order to guide an agent's reasoning, must be sensitive to which validity facts they think obtain (Steinberger 2019a, p. 317).

However, as Steinberger (2019a, pp. 323-324) acknowledges, the tripartite distinction cannot resolve the second conflict because both Preface Paradox and Strictness are relevant to the adequacy of directive validity bridge principles. An adequate directive validity bridge principle should not direct the author to either believe that there are no false sentences in her book or else forgo her belief in one of its claims, given that she is rationally required to believe both. Yet, an adequate directive validity bridge principle should direct agents to either believe the straightforward consequences of their belief sets or else forgo at least one of their existing beliefs. Thus, the space of directive validity bridge principles remains a conflict zone. Whether Harman's challenge can be overcome from the direction of validity bridge principles hinges on whether it can be pacified. ${ }^{15}$

To resolve the conflict between Strictness and Preface Paradox, Steinberger proposes that they are relevant to the adequacy of directive validity bridge principles in different contexts, depending on the number of premiss beliefs in play:

\footnotetext{
14 Steinberger (2019a, pp. 317-318; 2019c, pp. 28-29) highlights that many of Harman's respondentsincluding Field (2009), MacFarlane (2004), Milne (2009), and Streumer (2007)—have articulated validity bridge principles that cannot serve as the directives.

15 One way of satisfying both Preface Paradox and Strictness is to endorse a validity bridge principle which constrains agents' degrees of belief, as Field (2009) does. However, as Harman (1986, Ch. 3; 2009, pp. 334-335) highlights, a bridge principle constraining our degrees of belief would be too demanding to function as a directive norm, and therefore cannot meet his challenge.
} 
Our intuitions regarding Strictness apply in ordinary contexts, where the agent is dealing with a very limited number of beliefs and their manageable logical consequences. In situations like these, our Preface intuitions get no purchase on us... Our Preface intuitions only kick in once we deal with sufficiently large bodies of propositions...it is precisely in these contexts in which our Preface intuitions get a foothold that our intuitions regarding Strictness lose their force (Steinberger 2019a, pp. 323-324).

Since Strictness and Preface Paradox apply in different contexts, Steinberger concludes that the conflict between them is resolved. All that is required is the identification of a directive validity bridge principle which satisfies Strictness in few-premiss contexts and Preface Paradox in many-premiss contexts. In what follows I set aside the details of Steinberger's favoured directive validity bridge principles since we are only concerned with the tenability of a resolution built atop this distinction.

I contend that this proposed resolution falls short because there are many-premiss contexts in which Strictness applies and few-premiss contexts in which the rationale underpinning Preface Paradox applies. For a case of the first kind, suppose that, for all $n$ such that $1 \leq n \leq 10,000$, I believe that $n<(n+1)$, but I lack beliefs about whether $n<(n+i)$ when $i>1$. One day I discover that the 'greater than' relation is transitive which, together with my 9999 ' $n<(n+1)$ ' beliefs, entails that $1<10,000$. Despite this being a many-premiss context, Strictness applies: I am indefeasibly required to believe that $1<10,000$ rather than merely having a reason to do so.

A second and more intractable problem arises from the observation that the preoccupation with the preface paradox is unwarranted. The preface paradox creates problems for strict validity bridge principles which indefeasibly require agents to be logically consistent because other equally compelling epistemic norms require the author to have inconsistent beliefs. However, this phenomenon is not limited to many-premiss contexts. For instance, take the classic sceptical paradox:

(1) If you know that you are reading this paper and you know that reading this paper is incompatible with you being a brain-in-a-vat (BIV), then you know that you are not a BIV.

(2) You do not know you are not a BIV.

(3) You do not know that you are reading this paper.

As is the case in the preface paradox, compelling epistemic norms require you to believe both premisses and the negation of the paradoxical conclusion. And therefore, if the preface paradox suffices to show that there are many-premiss contexts in which an adequate validity bridge principle should not be strict-as Steinberger maintains it does - then the sceptical paradox suffices to show that there are also few-premiss contexts in which an adequate validity bridge principle should not be strict. That is, the rationale underpinning the Preface Paradox criterion actually extends far beyond many-premiss contexts. ${ }^{16}$

\footnotetext{
16 Harman (1986, pp. 15-17) makes a similar point in relation to the liar paradox, arguing that a bridge principle demanding consistency would be too strong: "To see that the Logical Inconsistency Principle has its exceptions, observe that sometimes one discovers that one's views are inconsistent and does not know how to revise them in order to avoid inconsistency without great cost. . So, the rational response [to the
} 
Since there are few-premiss contexts in which the rationale underpinning Preface Paradox applies and there are many-premiss contexts in which Strictness applies, distinguishing between these two kinds of contexts cannot resolve the conflict as Steinberger had hoped. For a validity bridge principle which satisfies Strictness in fewpremiss contexts will nonetheless contravene the motivations for Preface Paradox, and vice versa in many-premiss contexts. In neither context do we have a validity bridge principle which satisfies all the relevant adequacy criteria. Of course, this does not mean that the conflict cannot be resolved by drawing a distinction between different kinds of contexts - this remains an open question. However, it does mean that, at present, Conflict-Free* does not hold for the space of directive validity bridge principles. Thus, although Steinberger and others have made much progress, for the time being traditionalists are unable to overcome Harman's challenge by articulating directive validity bridge principles, and his challenge remains unmet. In what follows, I argue that invalidity bridge principles are up to the task.

\section{Invalidity bridge principles}

As we saw in Sect. 2, Harman's challenge may in principle be met by articulating an adequate directive invalidity bridge principle. The remainder of this paper proceeds as follows. This section provides a blueprint for invalidity bridge principles, thereby enabling us to generate the spaces of possible evaluative, directive, and appraisal invalidity bridge principles. Section 5 identifies the adequacy criteria relevant to each space, whilst Sects. 6 and 7 apply the criteria accordingly.

The validity bridge principles focussed on thus far capture only one way in which agents' reasoning can go wrong: namely, by failing to be sensitive to their belief sets' logical consequences. But there is another equally important way in which agents' reasoning can go wrong from a deductive standpoint: by reasoning invalidly. For instance, an agent who affirms the consequent by inferring $\varphi$ from $\varphi \supset \psi$ and $\psi$ has also reasoned incorrectly in an important sense. This is where invalidity bridge principles enter the fray.

At the most general level, invalidity bridge principles take the form:

Blueprint $_{1}$ : If $\delta(\Gamma \not \models \psi)$, then $N$.

As before, $\delta$ is a variable denoting a doxastic attitude which is empty when the antecedent is factual, whilst $N$ denotes the normative constraint on reasoning induced by the invalidity fact $\Gamma \not \psi$ or the agent's thinking that $\Gamma \not \psi$ obtains.

There are various kinds of constraints on reasoning which differ according to which aspect of reasoning they constrain. For validity bridge principles, $N$ is a normative constraint governing the combinations of beliefs an agent may have. However, unlike their validity counterparts, invalidity facts do not induce constraints on the combinations of

Footnote 16 continued

liar paradox] for most of us may simply be to recognise our beliefs about truth are logically inconsistent, agree this is undesirable, and try not to exploit the inconsistency in our inferences". 
beliefs agents may have. Given $\Gamma \models \varphi,\{\Gamma, \neg \varphi\}$ is inconsistent and so it is natural to think that, at the very least, agents are forbidden from believing $\Gamma \wedge \neg \varphi$. By contrast, given $\Gamma \not \forall \psi$ and $\Gamma \not \models \neg \psi$, neither $\{\Gamma, \psi\}$ nor $\{\Gamma, \neg \psi\}$ is inconsistent. Hence, from the standpoint of logic, it is permissible to have either combination of beliefs, which is to say that invalidity facts induce no substantive constraints on the combinations of beliefs that agents may have. ${ }^{17}$

If invalidity facts do not induce constraints on the combinations of beliefs one may hold, what kind of constraint do they induce? At a first pass, a natural answer is that the invalidity fact $\Gamma \not \forall \psi$ forbids one from forming the belief that $\psi$ via deduction from $\Gamma$ (the question of whether incorrect deduction should qualify as deduction in the first place is addressed in Sect. 6. For now I use 'deduction' as a placeholder). That is, rather than constraining which combinations of beliefs agents may have, the normative constraints induced by invalidity facts constrain the methods by which agents may form their beliefs. ${ }^{18}$ It is important to remember that methods are an aspect of reasoning, insofar as reasoning is the process of how we form and revise our beliefs. The articulation of an adequate bridge principle asserting that invalidity facts induce normative constraints on methods of belief formation would therefore suffice to show that logic is normative for reasoning.

Letting $M$ be a specification of the prohibited method of belief formation, this thought yields the following blueprint for invalidity bridge principles:

Blueprint 2 : If $\delta(\Gamma \not \psi)$, then $D \neg(M)$.

That is, if $\Gamma \not \forall \psi$, or $s$ thinks that $\Gamma \not \forall \psi$, then $s$ is forbidden from or has a reason against forming the belief that $\psi$ via method $M$. Invalidity bridge principles therefore admit of four dimensions of variation:

1. $\delta$ : Does the invalidity bridge principle have a factual antecedent in which $\delta$ is empty, or an attitudinal antecedent where $\delta$ denotes some attitude? That is, are agents subject to the normative constraints induced by the invalidity facts which actually obtain, or those that they think obtain?

2. D: Is the deontic operator 'ought to', 'has reason to', or 'is permitted to'?

3. $\neg$ 's Scope: Should $\neg$ take wide or narrow scope with respect to the deontic operator? That is, should an invalidity bridge principle's consequent be $\neg D(M)$, and assert it is not the case that $s$ ought to or has a reason to form the belief that $\psi$ by deduction from $\Gamma$ ? Or should it be $D \neg(M)$, and assert $s$ is forbidden from or has a reason against forming the belief that $\psi$ by deduction from $\Gamma$ ?

4. $M$ : How can we specify the method of belief formation proscribed by the normative constraints induced by invalidity facts?

By varying the values these four parameters take, we can generate the spaces of evaluative, directive, and appraisal invalidity bridge principles—albeit indeterminately

\footnotetext{
17 A natural thought is that invalidity facts do induce constraints on belief combinations: namely, if $\Gamma \not \notin \psi$ then one ought to believe that $\Gamma \not \models \psi$ and is forbidden from believing that $\Gamma \models \psi$. However, these are not logical norms governing combinations of beliefs, but instances of the truth and falsity norms for individual beliefs.

${ }^{18}$ For this reason, one cannot obtain invalidity bridge principles by negating both the antecedent and consequent of validity bridge principles.
} 
insofar as I have not catalogued the number of possible ways of specifying the prohibited method. However, this will suffice for now, and we turn to the criteria relevant to the adequacy of invalidity bridge principles.

\section{Adequacy criteria for invalidity bridge principles}

This section identifies the adequacy criteria relevant to invalidity bridge principles and ascertains which of these criteria are relevant to the spaces of evaluative, directive, and appraisal invalidity bridge principles. A natural starting point is with the adequacy criteria pertaining to validity bridge principles - though given that validity and invalidity bridge principles induce different kinds constraints on reasoning, we should expect different sets of adequacy criteria to apply to them. We consider each of MacFarlane's adequacy criteria in turn.

(1) Belief Revision. Since invalidity bridge principles merely require that one not form certain beliefs in a particular way, there is no worry that they might require one to believe the consequences of one's existing belief set regardless of their plausibility.

(2) Excessive Demands. Can it be over-demanding to forbid agents from forming beliefs in a particular way? Invalidity bridge principles can be over-demanding because it can be difficult to determine whether $\Gamma \not \forall \psi$ and therefore which beliefs cannot be formed via the proscribed method. Moreover, even if one need not have ascertained all the propositions not entailed by $\Gamma$ to not invalidly deduce them from $\Gamma$, invalidity bridge principles might be over-demanding in another way. Since we are liable to reasoning invalidly, it may be over-demanding to require that agents not form the belief that $\psi$ via deduction from $\Gamma$ when they are unaware that $\Gamma \not \models \psi$.

(3) Preface Paradox. Adhering to invalidity bridgeprinciples does not require one to believe the logical consequences of one's beliefs, and therefore abiding by them does not force the author to having to forgo either of her rationally required but inconsistent beliefs.

(4) Clutter Avoidance. Since invalidity bridge principles do not require one to hold any particular beliefs, abiding by them cannot result in a cluttered belief set.

(5) Priority Question. This criterion asserts that the normative constraints on reasoning induced by logic bind us irrespective of whether we think the logical facts which induce them obtain. Accordingly, this criterion applies equally to invalidity bridge principles: the normative constraints induced by invalidity facts constrain our reasoning irrespective of whether we think these facts obtain.

(6) Strictness. This criterion asserts the normative constraints on reasoning induced by logic should be indefeasible, and therefore also applies to invalidity bridge principles because one ought not deduce $\psi$ from $\Gamma$ if one thinks that $\Gamma \not \forall \psi$.

(7) Obtuseness. This criterion demands that adequate bridge principles positively require agents to believe their belief sets' consequences rather than merely forbidding them from disbelieving their beliefs' consequences. Since invalidity bridge principles do not concern which beliefs agents have but how they are formed, Obtuseness does not apply to them. 
Accordingly, of the criteria which applied to validity bridge principles, three apply to their invalidity cousins. ${ }^{19}$ Are there additional criteria an adequate invalidity bridge principle must satisfy? On the face of it, there is at least one more:

(8) Alternative Methods. Although $\Gamma \not \forall \psi$ induces a normative constraint forbidding agents from forming the belief that $\psi$ via deduction from $\Gamma$, this constraint should not forbid agents from forming the belief that $\psi$ via alternative methods-in particular, via non-deductive inference from $\Gamma$. For instance, 'The sun will rise tomorrow morning' is not entailed by 'The sun has risen every previous morning', but an adequate invalidity bridge principle must not forbid agents from forming a belief in the former via inductive inference from the latter.

Thus we have four adequacy criteria: Priority Question, Excessive Demands, Strictness, and Alternative Methods. Recall from Sect. 3 that bridge principles are norms, and norms can perform evaluative, directive, or appraisal functions. Accordingly, there is not one space of invalidity bridge principles but three, and to ascertain whether there are any invalidity bridge principles satisfying these criteria we must first clarify which criteria apply to the different spaces.

We begin with Priority Question, which asserts that the normative constraints on reasoning induced by invalidity facts constrain our reasoning irrespective of whether we think that the invalidity facts in question obtain. As was the case for validity bridge principles, whilst this is appropriate for evaluative invalidity bridge principles which are concerned with objective and idealised standards for reasoning, it is inappropriate for directive invalidity bridge principles which guide agents' reasoning. Turning to appraisal invalidity bridge principles, should agents be reprimanded for being insensitive to invalidity facts they are unaware of? (Steinberger 2019c, p. 21) reminds us that criticisms of an agent's reasoning need not be based on the invalidity facts the agent thinks obtain: agents' reasoning can also be criticised for contravening the normative constraints induced by the invalidity facts that they are reasonably expected to recognise but do not. Moreover, which facts agents can be reasonably expected to recognise varies across contexts. In low idealisation contexts, such as informal discussion, an agent's reasoning cannot be criticised for failing to respond to an invalidity fact they are unaware of. By contrast, in higher idealisation contexts, such as a logic seminar, an agent can be criticised for failing to be sensitive to the same invalidity fact, despite still being unaware of it. Thus, Priority Question is relevant to the adequacy of appraisal invalidity bridge principles in high but not low idealisation contexts (Steinberger 2019c, p. 27).

Second, Excessive Demands, according to which an adequate invalidity bridge principle should not require agents to do what lies beyond their cognitive capacities. Since evaluative invalidity bridge principles concern ideal standards, they abstract away from agents' cognitive limitations and therefore do not lie within Excessive Demands' jurisdiction. By contrast, Excessive Demands is relevant to directive validity bridge principles because, if they are to guide our reasoning, they cannot require that we do what we are incapable of. Finally, Excessive Demands applies to appraisal invalidity bridge principles in low idealisation contexts since part of what it is for

\footnotetext{
19 This is perhaps unsurprising given that Belief Revision, Clutter Avoidance, and Paradox are all criticisms levelled by Harman against a validity bridge principle which requires agents to believe all the logical consequences of their belief sets.
} 
an appraisal norm to be suitable for a low idealisation context is that it is not overdemanding. Since these worries have no place in high idealisation contexts, Excessive Demands does not apply.

Our third adequacy criterion, Strictness, is relevant to all three kinds of norms. Strictness is relevant to evaluative invalidity bridge principles because part of the very notion of an objective evaluative ideal is that it is strict (Steinberger 2019c, p. 26). It is also relevant to directive invalidity bridge principles because, at least in cases where the agent thinks an invalidity fact obtains, an adequate invalidity bridge principle should strictly forbid the agent from performing the incorrect deduction.

With appraisal invalidity bridge principles matters are slightly more complex, and it is worth beginning by explicitly stating how the choice of deontic operator affects an appraisal invalidity bridge principle. If one violates an appraisal norm, then one is liable to criticism. Thus, if an appraisal norm employs the indefeasible 'ought to' operator then, provided the triggering condition is met, failure to comply is always reprehensible. By contrast, if an appraisal norm employs the defeasible 'has reason to' operator, then whether an agent's non-compliance is reprehensible depends on whether the reason provided by the norm for doing as it says 'outweighs' the agent's reasons for not doing so. If it does then $s$ is reprehensible, and vice versa. Accordingly, in the context of appraisal invalidity bridge principles, Strictness asserts that, at least when it comes to straightforward invalidity facts that the agent thinks obtain, agents are criticisable if they contravene the normative constraints induced by said facts, irrespective of their reasons for doing so. And this seems correct. From the standpoint of logic, if an agent thinks that $\varphi \vee \psi \not \forall \psi$ but then invalidly infers $\psi$, their conduct is criticisable irrespective of their reasons for doing so.

We may also ask whether Strictness applies differently between low and high idealisation contexts. In other words, does which invalidity facts $s$ is expected to recognise cause whether or not $s$ is criticisable for violating the corresponding normative constraints to be sensitive to $s$ 's reasons for contravening them? For my part, I cannot see why this would be so. The fact that $s$ is in a low idealisation context has already been accounted for by the endorsement of an appraisal invalidity bridge principle with an attitudinal antecedent. To weaken the deontic operator in addition would be, in effect, to double count when there seems little reason to do so. Put another way, idealisation affects which invalidity facts an agent can be reasonably expected to recognise. Irrespective of which invalidity facts an agent can be reasonably expected to recognise, if it is genuinely reasonable to expect them to do so, they are always reprehensible if they contravene the corresponding normative constraints. Thus, Strictness is not sensitive to the appropriate degree of idealisation.

Finally, Alternative Methods. Suppose that $\Gamma \not \psi$ and $\Gamma$ provides non-deductive support for $\psi$. It is not incorrect for $s$ to form the belief that $\psi$ via non-deductive inference from $\Gamma$; and $s$ should be neither directed not to do so nor criticised for doing so. Thus, Alternative Methods applies to all three spaces.

These findings are summarised in the Table 1, in which the asterisks denote the fact that the extent to which the adequacy criterion applies to that kind of norm depends upon the degree of idealisation at play. Having identified the adequacy criteria pertaining to invalidity bridge principles, we may now begin the task of ascertaining which permutations of our invalidity bridge principle blueprint, if any, satisfy them. 
Table 1 Adequacy criteria for invalidity bridge principles

\begin{tabular}{llll}
\hline Criterion & Evaluations & Directives & Appraisals \\
\hline Priority question & $\checkmark$ & $x$ & $\checkmark *$ \\
Excessive demands & $x$ & $\checkmark$ & $\checkmark *$ \\
Strictness & $\checkmark$ & $\checkmark$ & $\checkmark$ \\
Alternative methods & $\checkmark$ & $\checkmark$ & $\checkmark$ \\
\hline
\end{tabular}

\section{The prohibited method}

The previous two sections have specified the dimensions along which invalidity bridge principles may vary and the criteria for assessing the adequacy of these permutations. We begin the task of ascertaining whether there are any adequate invalidity bridge principles with the specification of the method of belief formation prohibited by the normative constraints induced by invalidity facts.

Given that $\Gamma \not \psi \psi$, an intuitive idea is to forbid $s$ from believing that $\psi$ because they believe $\Gamma$. Let $\sqsupset$ denote the 'in virtue of' relation such that $p \sqsupset q$ reads 'In virtue of $p, q$ ', and let $B$ be the binary predicate '...believes that...'. We may then capture this suggestion as follows:

$$
I B P_{1}: \text { If } \delta(\Gamma \not \psi) \text {, then } D \neg(B s \Gamma \sqsupset B s \psi) \text {. }
$$

However, this proposal is inadequate. An agent who forms the belief that the sun will rise tomorrow morning via the method of inductive inference from the sun has risen every previous morning believes the former in virtue of the latter. Therefore, in violation of Alternative Methods, $\mathrm{IBP}_{1}$ forbids agents from forming the belief that the sun will rise tomorrow via induction. ${ }^{20}$

Perhaps a bid to avoid this issue, Steinberger's (2019b, p. 5) proposal distinguishes between logical and non-logical normative reasons for belief, where one has a logical reason to believe $\psi$ iff $\psi$ is entailed by one's belief set. Let $R$ be the binary predicate '...has a non-logical reason to believe...' and $P$ be the 'is permitted to' operator. Steinberger endorses the following invalidity bridge principle:

$$
I B P_{2}: \text { If } \delta(\Gamma \forall \psi) \wedge \neg R s \psi \text {, then } \neg(P(B s \Gamma) \supset P(B s \psi)) .{ }^{21}
$$

That is, if ( $s$ thinks that) $\Gamma \not \psi \psi$ and $s$ has no non-logical reason to believe $\psi$, then it is not the case that if $s$ is permitted to believe $\Gamma$ then $s$ is permitted to believe $\psi$. The

\footnotetext{
${ }^{20}$ A seemingly plausible variant of $I B P_{1}$ is: If $\delta(\Gamma \not \models \psi)$, then $D \neg(B s(\Gamma \wedge(\Gamma \models \psi)) \sqsupset B s(\psi))$. That is, if $\Gamma \not \forall \psi$, or $s$ thinks $\Gamma \not \forall \psi$, then $s$ ought not (or has reason not to) believe $\psi$ because they believe $\Gamma$ and $\Gamma \models \psi$. However, it is difficult to see how such a principle could function as a directive norm. To be a directive norm, its antecedent must be attitudinal, yielding: If $B s(\Gamma \not \forall \psi)$, then $D \neg(B s(\Gamma \wedge(\Gamma \models \psi)) \sqsupset B s(\psi))$. However, unless $s$ believes both $\Gamma \not \psi \psi$ and $\Gamma \models \psi$, this constraint is vacuous. If we try and avoid this by removing the occurrence of $\Gamma \models \psi$ in the consequent-so it reads: If $B s(\Gamma \not \forall \psi)$, then $D \neg(B s \Gamma \sqsupset B s(\psi))$ 一 we are back to $I B P_{1}$.

${ }^{21} I B P_{2}$ is the contraposition of the invalidity bridge principle Steinberger endorses.
} 
distinction between logical and non-logical reasons for belief enables $I B P_{2}$ to parry the induction example because this is not a case in which $s$ has no non-logical reason to believe $\psi$. What is important for present purposes is neither Steinberger's choice of deontic operator nor its scope, but the general strategy of adding the 'and $s$ has no non-logical reason for believing $\psi$ ' clause to the antecedent.

This strategy renders $I B P_{2}$ susceptible to two shortcomings. First, $I B P_{2}$ is not a constraint induced by invalidity facts. Given that: (1) the distinction between logical and non-logical reasons partitions the space of normative reasons for belief, and (2) it is a necessary condition for one's having a logical reason to believe $\psi$ that one's existing belief set entails $\psi$; it follows that $I B P_{2}$ 's antecedent simply asserts $s$ has no reason to believe $\psi$. Accordingly, $I B P_{2}$ asserts: if $s$ has no normative reason to believe $\psi$, then it is not the case that if $s$ is permitted to believe $\Gamma$ then $s$ is permitted to believe $\psi$. However, supposing $s$ is permitted to believe $\Gamma$, that $s$ is not permitted to believe what they have no normative reason to believe is not a constraint induced by invalidity facts. ${ }^{22}$ Rather, it is a more general constraint, perhaps related to the principle of underdetermination familiar from epistemology.

The second problem is that, by incorporating the 'and $s$ has no non-logical reason for believing $\psi$ ' clause into $I B P_{2}$ 's triggering condition, agents are not constrained by the normative constraint given in $I B P_{2}$ 's consequent whenever they have non-logical reasons for believing $\psi$. But this is incorrect: even if I have non-logical reasons for believing that Hypatia is a woman, I am still forbidden from deducing this from the propositions that Hypatia is mortal and that if Hypatia is a woman then she is mortal. Thus, $I B P_{2}$ may be rejected because it states a constraint not induced by invalidity facts and it does not constrain agents' reasoning when they have non-logical normative reasons for believing $\psi$.

Where do we go from here? I endorse the following proposal. Let $F$ be the tertiary predicate '...forms the belief that...via method...' and $m$ be the method of forming a belief via deduction from $\Gamma$. We can articulate the following constraint:

$$
I B P_{3}: \text { If } \delta(\Gamma \not \forall \psi) \text {, then } D \neg(F s \psi m) \text {. }
$$

That is, if $\Gamma \nvdash \psi$, or $s$ thinks $\Gamma \not \psi$, then $s$ is forbidden from or has a reason against forming the belief that $\psi$ via deduction from $\Gamma .{ }^{23}$ The crucial issue here is how the method of belief formation I have been referring to using 'deduction' is individuated in $I B P_{3}$. When individuating deduction-and methods of belief formation more generallyone may do so either infallibly or fallibly. On an infallibilist individuation of deduction, only valid inferences qualify as tokens of deduction and therefore any belief formed via this method is true conditional on the premiss beliefs being true. By contrast, on an infallibilist individuation of deduction, instances of invalid deduction may qualify as tokens of deduction. For $I B P_{3}$ to get off the ground, deduction must be individuated fallibly. This is because if deduction were individuated infallibly, so that only forming

\footnotetext{
22 One might doubt whether this is a constraint at all. For instance, agents might be permitted to believe something when they have a suitable motivating reason which fails to be normative.

23 One can obtain a substantive invalidity bridge principle employing the 'permitted to' operator by adopting a principle in which $\neg$ has a wide scope. The resulting principle forbids agents from forming the belief that $\psi$ via deduction from $\Gamma$.
} 
beliefs via valid inferences qualified as tokens of deduction, then $I B P_{3}$ would be vacuous as it would forbid agents from doing the impossible: namely, forming the belief that $\psi$ via a valid inference from $\Gamma$.

Luckily, fallibilist method individuation is a mainstream position in contemporary epistemology — endorsed by, inter alia, Becker (2012), Brown (2018), Goldman (1979), Nozick (1981), and Sosa (1991)—and there are strong independent arguments in its favour. To give just two examples, Sosa (1991, Ch. 13) argues against infallibilist individuations because they divorce methods' reliability from ordinary agents' cognitive capacities, and his argument applies equally to infallibilist individuations of deduction. To see how, suppose that Ruth is an impeccable logician whilst Jacques is deductively inept. On an infallibilist individuation of deduction, none of the instances in which Jacques reasons invalidly qualify as tokens of deduction, and therefore the reliability of forming beliefs via deduction for him is unattenuated by his deductive incompetence. Thus, despite Ruth's proficiency and Jacques' ineptitude, individuating deduction infallibly entails that forming beliefs via deduction is an equally reliable method of belief formation for the two of them, which Sosa concludes is "absurd" (1991, p. 234). ${ }^{24}$ Elsewhere, Brown (2018, pp. 114-119) has argued that infallibilist individuations ought to be rejected because they are incompatible with the widely accepted phenomenon of epistemic defeat, where a belief loses a status like justification. After all, how could one lose justification for a belief produced via an infallible method? Applying this to deduction, an infallibilist individuation wrongly entails that a justified belief produced via deduction could never cease to be justified, irrespective of what evidence to the contrary the agent may acquire.

It is also straightforward to see that $I_{B P}$ also satisfies Alternative Methods when deduction is individuated fallibly because any plausible fallibilist individuation found in the epistemlogy literature will not count instances of forming beliefs via nondeductive methods as tokens of deduction. I therefore remain neutral on exactly how deduction is individuated, and invite the reader to plug in their preferred fallibilist individuation. ${ }^{25}$ Having clarified how the prohibited method of belief formation is to be specified, we may now turn to the question of whether there are any variants of $I B P_{3}$ satisfying the remaining adequacy criteria.

\section{Are there any adequate invalidity bridge principles?}

We are finally ready to ascend the summit and ascertain whether there are any adequate evaluative, directive, or appraisal invalidity bridge principles. I contend there are adequate invalidity bridge principles of all three kinds. I begin by reiterating which

\footnotetext{
24 Sosa also highlights that infallibilist individuations cannot accommodate the fact that "a logician gifted with excellent deductive powers who goes through a relatively simple proof and somehow fails for once to detect an invalidating flaw... [is] justified still in believing the (false) conclusion on the basis of his inference from the (true) premises" (1991, p. 232).

25 I favour Sosa's (1991) individuation on which a belief is formed via deduction when it is produced by the faculty of deduction. The existence of a distinct deductive faculty is corroborated by recent findings in neuropsychology—see Coetzee and Monti (2018).
} 
values of $I B P_{3}$ 's parameters Priority Question, Excessive Demands, and Strictness favour, and then apply them to the three spaces of invalidity bridge principles.

Priority Question favours a factual antecedent in order to reflect the fact that we are subject to the normative constraints induced by invalidity facts irrespective of whether we think that these facts obtain. By contrast, to avoid being over-demanding, Excessive Demands favours an attitudinal antecedent so that agents are only subject to the normative constraints induced by invalidity facts that they think obtain. Lastly, Strictness is doubly relevant to invalidity bridge principles. First, as before, it recommends that the deontic operator be 'ought to'. Second, Strictness favours the negation taking narrow scope with respect to the deontic operator so that agents are forbidden from deducing $\psi$ from $\Gamma$ when $\Gamma \not \forall \psi$, rather than the normative constraint merely saying it is not the case that agents ought to deduce $\psi$ from $\Gamma$.

A crucial point here is that although Priority Question and Excessive Demands require the antecedent's attitudinal parameter to take different values, this conflict is easily dissipated. There is no conflict with respect to evaluative and directive invalidity bridge principles since, as highlighted in Sect. 5, it is not the case that both criteria apply to either of these spaces. However, both Priority Question and Excessive Demands apply to appraisal invalidity bridge principles. This conflict is defused by the distinction between low and high idealisation contexts. In low idealisation contexts, where agents cannot be reasonably expected to abide by the normative constraints induced by invalidity facts of which they are unaware, Excessive Demands applies but Priority Question does not, and the reverse holds in high idealisation contexts. ${ }^{26}$ Thus, this distinction ameliorates the conflict and the possibility of there being adequate invalidity bridge principles of all three kinds remains open.

Let us begin with evaluative invalidity bridge principles. Since only Priority Question and Strictness apply to them, an adequate evaluative invalidity principle must have a factual antecedent, its deontic operator must be 'ought to' $(O)$, and the negation must take narrow scope with respect to this operator. Thus, the following is an adequate evaluative invalidity bridge principle:

$$
I B P_{E}: \text { If } \Gamma \not \psi \text {, then } O \neg(F s \psi m) \text {. }
$$

That is, if $\Gamma$ does not entail $\psi$, then $s$ is forbidden from forming the belief that $\psi$ via deduction from $\Gamma$.

With directive invalidity bridge principles, matters are somewhat different. Priority Question is irrelevant to directives, whilst Excessive Demands and the normative function of directive invalidity bridge principles both dictate that their triggering conditions be cognitively accessible to agents and therefore that their antecedents be attitudinal. This still leaves open the question of what attitude $\delta$ should be. As highlighted by Steinberger (2019c, p. 20), one problem with 'believes' is that very often agents will lack explicit beliefs about invalidity facts-perhaps because they lack the requisite concepts - and therefore only those who have such beliefs will be bound by logic. To

\footnotetext{
26 In effect, we can think of there as being two spaces of appraisal invalidity bridge principles, a low idealisation space and a high idealisation space, and whereas Excessive Demands applies to the low but not the high idealisation space, the reverse is true of Priority Question. Since they do not apply to the same space, the conflict is dissolved.
} 
avoid this unwelcome intellectualism, $\delta$ should be some kind of attitude that does not presuppose explicit belief or possession of the requisite concepts. For convenience I follow Steinberger in opting for 'In $s$ 's best estimation...'. Since Strictness applies as it did in the case of evaluative invalidity bridge principles, the following permutation satisfies all the relevant adequacy criteria:

$I B P_{D}$ : If in $s$ 's best estimation $\Gamma \not \forall \psi$, then $O \neg(F s \psi m)$.

This asserts that, if in $s$ 's best estimation $\Gamma$ does not entail $\psi$, then $s$ is forbidden from forming the belief that $\psi$ via deduction from $\Gamma$.

Finally, we turn to appraisal invalidity bridge principles. Strictness is relevant to appraisal invalidity bridge principles and applies in the usual way. However, as argued in Sect. 5, whether Excessive Demands and Priority Question are relevant to the adequacy of appraisal invalidity bridge principles is context-dependent. Instead of endorsing distinct appraisal invalidity bridge principles for different contexts, we can endorse the following permutation whose antecedent automatically adjusts itself to the degree of idealisation appropriate to a particular context:

$I B P_{A}$ : If $s$ ought to recognise that $\Gamma \not \forall \psi$, then $O \neg(F s \psi m)$.

That is, if $s$ ought to recognise that $\Gamma \not \models \psi$, then $s$ is forbidden from forming the belief that $\psi$ via deduction from $\Gamma .^{27}$

In low idealisation contexts where $s$ should only be reprimanded for violating normative constraints induced by the invalidity facts they believe to obtain, $I B P_{A}$ 's antecedent effectively reduces to $B s(\Gamma \not \forall \psi)$, thus satisfying Excessive Demands. By contrast, in high idealisation contexts where $s$ is expected to recognise every invalidity fact and abide by the normative constraints they induce, $I B P_{A}$ 's antecedent can be read as $\Gamma \nvdash \psi$, thereby satisfying Priority Question. At intermediate levels of idealisation, agents are expected to abide by the normative constraints induced by invalidity facts beyond those they believe but not by every invalidity fact. Accordingly, we can understand $I B P_{A}$ 's antecedent as reducing to a claim specifying some relation between $s$ and invalidity facts which $s$ bears to a wider range of invalidity facts than those they believe but not to all invalidity facts-such as 'In $s$ 's best estimation'. Thus, in $I B P_{A}$ we have an appraisal invalidity bridge principle which satisfies all the relevant adequacy criteria.

Since $I B P_{E}, I B P_{D}$, and $I B P_{A}$ all satisfy the adequacy criteria pertaining to them, it follows there are adequate invalidity bridge principles of all three kinds. That is, logic is normative in all three senses: it provides evaluative norms stating ideal standards of correctness for reasoning, directive norms telling us how to reason, and appraisal norms delineating when our reasoning is reprehensible or praiseworthy.

\footnotetext{
27 One worry with all three proposed invalidity bridge principles is that they incorrectly forbid agents from forming beliefs via non-logical deductions. For instance, 'A is taller than $B$ ' and 'B is taller than $C$ ' do not entail that 'A is taller than C', but agents should not be forbidden from deducing the latter from the former. I think that this potentially serious worry can be avoided because arguments such as the one above are most plausibly understood as enthymemes. Once the tacit premiss is acknowledged-in this case, 'If A is taller than $\mathrm{B}$ and $\mathrm{B}$ is taller than $\mathrm{C}$, then $\mathrm{A}$ is taller than $\mathrm{C}^{\prime}$ - the premisses entail the conclusion, and so agents are not forbidden from deducing the conclusion from the premisses. Thanks to an anonymous referee for raising this point.
} 
We may now return to Harman's challenge. Harman observed that logic is normative for reasoning iff there is an adequate directive bridge principle. His challenge to traditionalists was to identify such a principle. Harman himself argued that no directive bridge principle satisfied all of the relevant adequacy criteria, and so concluded that logic is not normative for reasoning. However, if the foregoing arguments are sound, then his modus tollens can be turned into a modus ponens: logic is normative for reasoning because there is an adequate directive bridge principle, perhaps just not of the kind originally envisaged.

\section{Conclusion}

According to Harman's sceptical challenge, logic is not normative for reasoning in the sense of providing norms that guide agents' reasoning - that is, there are no adequate directive bridge principles. This paper has identified a new class of bridge principles, invalidity bridge principles, and argued they can be used to successfully rebut Harman's challenge.

We began with the observation that Harman's challenge can be surmounted from either of two directions: by articulating an adequate directive validity bridge principle or an adequate directive invalidity bridge principle. Although traditionalists have exclusively attempted to surmount Harman's challenge from the first direction, I argued that this strategy cannot succeed for the time being. The reason for this was that the conflict between the Strictness and Preface Paradox adequacy criteria has yet to be satisfactorily resolved, which leaves traditionalists unable at the present time to articulate a directive validity bridge principle that satisfies all the relevant adequacy criteria. This motivated approaching Harman's challenge from the direction of invalidity bridge principles. I argued that invalidity facts such as $\Gamma \not \psi$ induce normative constraints forbidding agents from forming the belief that $\psi$ via deduction from $\Gamma$. I then demonstrated that not only can one successfully surmount Harman's challenge from this direction since there is an adequate directive invalidity bridge principle, but that there are also adequate evaluative and appraisal invalidity bridge principles. Thus, traditionalists can rest easy: logic is normative for reasoning after all.

Acknowledgements Thanks go to the two anonymous referees who took the time to provide constructive comments, and the audience at the 13th Annual Cambridge Graduate Conference on the Philosophy of Mathematics \& Logic; this paper has improved significantly as a result of their input. I'm extremely grateful to my supervisors, Franz Berto and Kevin Scharp, for their feedback and, especially, for their encouragement. Finally, since this is my first publication, I'd like to thank my mum for her unwavering support.

Open Access This article is licensed under a Creative Commons Attribution 4.0 International License, which permits use, sharing, adaptation, distribution and reproduction in any medium or format, as long as you give appropriate credit to the original author(s) and the source, provide a link to the Creative Commons licence, and indicate if changes were made. The images or other third party material in this article are included in the article's Creative Commons licence, unless indicated otherwise in a credit line to the material. If material is not included in the article's Creative Commons licence and your intended use is not permitted by statutory regulation or exceeds the permitted use, you will need to obtain permission directly from the copyright holder. To view a copy of this licence, visit http://creativecommons.org/licenses/by/4.0/. 


\section{References}

Beall, J. C., \& Restall, G. (2006). Logical pluralism. Oxford: Clarendon Press.

Becker, K. (2012). Methods and how to individuate them. In K. Becker \& T. Black (Eds.), The sensitivity principle in epistemology (pp. 81-97). Cambridge: Cambridge University Press.

Brown, J. (2018). Fallibilism: Evidence and knowledge. Oxford: Oxford University Press.

Coetzee, J. P., \& Monti, M. M. (2018). At the core of reasoning: Dissociating deductive and non-deductive load. Human Brain Mapping, 39, 1850-1861.

Dogramaci, S. (2015). Communist conventions for deductive reasoning. Nô̂s, 49, 776-799.

Dutilh-Novaes, C. (2015). A dialogical, multi-agent account of the normativity of logic. Dialectica, 69, 587-609.

Field, H. (2009). The normative role of logic. Proceedings of the Aristotelian Society, 83, 251-268.

Frege, G. (1893). The basic laws of arithmetic (P. A. Ebert \& M. Rossberg, Eds.). Oxford: Oxford University Press.

Goldman, A. I. (1979). What is justified belief? In G. S. Pappas (Ed.), Justification and knowledge (pp. 1-23). Dordrecht: Reidel.

Harman, G. H. (1984). Logic and reasoning. Synthese, 60, 107-127.

Harman, G. H. (1986). Change in view: Principles of reasoning. Cambridge, MA: MIT Press.

Harman, G. H. (2009). Field on the normative role of logic. Proceedings of the Aristotelian Society, 109, $333-335$.

Harman, G. H. (2010). Epistemology as methodology. In J. Dancy, E. Sosa, \& M. Steup (Eds.), A companion to epistemology (2nd ed., pp. 152-156). Malden, MA: Wiley-Blackwell.

Kant, I. (1787). Critique of Pure Reason (M. Weigelt, Ed. \& M. Müller, Trans.). London: Penguin.

Kant, I. (1885). Kant's introduction to logic and his essay on the mistaken subtility of the four figures (T. K. Abbott, Trans.). London: Longmans, Green, \& Co.

MacFarlane, J. (2004). In what sense (if any) is logic normative for thought? Unpublished Manuscript.

Makinson, D. C. (1965). The Paradox of the preface. Analysis, 25, 205-207.

Milne, P. (2009). What is the normative role of logic? Proceedings of the Aristotelian Society, 83, $269-298$.

Nozick, R. (1981). Philosophical explanations. Cambridge, MA: Harvard University Press.

Pinder, M. (2017). A normative argument against explosion. Thought, 6, 61-70.

Priest, G. (1979). Two dogmas of quineanism. Philosophical Quarterly, 29, 289-301.

Russell, G. (2020). Logic isn't normative. Inquiry, 63, 371-388.

Smith, R. (1995). Logic. In J. Barnes (Ed.), The Cambridge companion to Aristotle (pp. 27-65). Cambridge: Cambridge University Press.

Sosa, E. (1991). Knowledge in perspective. Cambridge: Cambridge University Press.

Steinberger, F. (2019a). Consequence and Normative Guidance. Philosophy and Phenomenological Research, 98, 306-328.

Steinberger, F. (2019b). Logical pluralism and logical normativity. Philosophers' Imprint, 19, 1-19.

Steinberger, F. (2019c). Three ways in which logic might be normative. Journal of Philosophy, 116, 5-31.

Streumer, B. (2007). Reasons and entailment. Erkenntnis, 66, 353-374.

Publisher's Note Springer Nature remains neutral with regard to jurisdictional claims in published maps and institutional affiliations. 